\title{
A multi-color fast-switching microfluidic droplet dye laser $\dagger$
}

\author{
Sindy K. Y. Tang, ${ }^{a}$ Zhenyu Li, $^{b}$ Adam R. Abate,${ }^{c}$ Jeremy J. Agresti, ${ }^{c}$ David A. Weitz, ${ }^{c}$ Demetri Psaltis ${ }^{d}$ \\ and George M. Whitesides*a
}

\author{
Received 14th July 2009, Accepted 10th August 2009 \\ First published as an Advance Article on the web 14th August 2009 \\ DOI: $10.1039 / b 914066 b$
}

\begin{abstract}
We describe a multi-color microfluidic dye laser operating in whispering gallery mode based on a train of alternating droplets containing solutions of different dyes; this laser is capable of switching the wavelength of its emission between $580 \mathrm{~nm}$ and $680 \mathrm{~nm}$ at frequencies up to $3.6 \mathrm{kHz}$ - the fastest among all dye lasers reported; it has potential applications in on-chip spectroscopy and flow cytometry.
\end{abstract}

We describe a microfluidic droplet dye laser that is capable of switching the wavelength of its emission at frequencies up to $3.6 \mathrm{kHz}$. This laser is based on a train of alternating droplets $(20-40 \mu \mathrm{m}$ in diameter) containing solutions of different dyes - each capable of lasing at a different wavelength - suspended in a fluorocarbon carrier liquid, in a polydimethylsiloxane (PDMS) microchannel. These drops flow through a region where the dyes in the drops are excited optically — one at a time - and lase. The dye being excited determines the lasing wavelength. Lasing occurs in whispering gallery modes: ${ }^{1-3}$ the drops, which have an index of refraction higher than that of the carrier liquid, are optical microcavities; light emitted from the dyes is confined and amplified in the drops by total internal reflection. Minimization of surface energy between the drop and the carrier fluid causes the surface of the drop to be optically smooth; optical loss due to scattering by the surface is, therefore, minimal.

The main advantage of this system is the capability for high-speed generation and switching of droplets containing different dyes (frequencies up to $100 \mathrm{kHz}$, or switching times of 10 microseconds) ) $^{4,5}$ over a large spectral range $(>100 \mathrm{~nm})$. The switching times of other microfluidic dye lasers are typically more than hundreds of milliseconds, since these lasers are usually limited by mass transport (different solutions of dyes must be injected into the region where optical pumping occurs to produce a change in lasing wavelength ${ }^{6,7}$ ), or by the speed of mechanical actuation. ${ }^{8}$ The rapid switching between lasing wavelengths that we describe here has the potential to be useful for high-throughput on-chip spectroscopy, and for flow cytometry; in both applications, multiple lasing (or amplified stimulated emission) frequencies are necessary for the excitation of different fluorescent labels. ${ }^{9}$

${ }^{a}$ Department of Chemistry and Chemical Biology, Harvard University, Cambridge, MA, 02138, USA. E-mail: gwhitesides@gmwgroup.harvard. edu; Fax: +1-617-495-9857; Tel: +1-617-495-9430

${ }^{b}$ Department of Electrical Engineering, California Institute of Technology, Pasadena, CA, 91125, USA

'School of Engineering and Applied Sciences/Department of Physics, Harvard University, Cambridge, MA, 02138, USA

${ }^{d}$ Optics Laboratory, School of Engineering, Swiss Federal Institute of Technology Lausanne (EPFL), CH-1015 Lausanne, Switzerland

$\dagger$ Electronic supplementary information (ESI) available: Fig. S1, Fig. S2 and a discussion about lasing modes. See DOI: 10.1039/b914066b
We fabricated the microfluidic channel in PDMS using conventional soft lithography. ${ }^{10}$ The microfluidic system has two functional parts: droplet generators and region for optical excitation (Fig. 1). For simplicity, we caused the droplets to be generated in alternation, rather than switching them actively, but active switching is a known methodology at frequencies up to $4 \mathrm{kHz} .{ }^{11} \mathrm{We}$ used two T-junctions that share the same main channel for the generation of droplets containing two different solutions of dyes. The alternation of droplets from these two opposing $\mathrm{T}$-junctions was spontaneous at the rates of flow that we were using: ${ }^{12,13}$ when a drop was forming from one $\mathrm{T}$-junction, the resistance of the main channel increased, and hindered the advance of the fluid thread from the other T-junction. The pinch-off of the fluid thread from the T-junction accelerated the advance of the fluid in the opposing T-junction. The fluid threads from the two T-junctions therefore broke up in alternation.

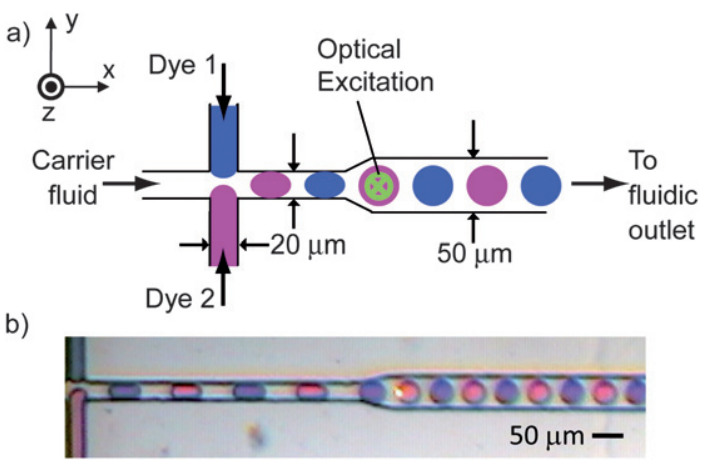

Fig. 1 a) shows a schematic diagram of the droplet laser. It consisted of two opposing T-junctions, and allowed spontaneous generation of droplets containing dyes 1 and 2 in alternation. The width of the channels at the T-junctions was $20 \mu \mathrm{m}$. The channel expanded in width to $50 \mu \mathrm{m}$ downstream. The height of the channel (in the z-direction) was $40 \mu \mathrm{m}$. The droplets were optically excited downstream by a frequency-doubled $\mathrm{Nd}$ :YAG directed perpendicular to the plane of the microchannel (in the z-direction). The optical output from the drops was collected through an objective behind the channel in the same direction as the pump beam (z-direction). See Fig. S1 for details on setup. $\dagger$ b) shows a snapshot of the device generating drops containing rhodamine 560 (pink) and oxazine 720 (purple) respectively, from a movie recorded with a colored chargecoupled device (CCD) camera. The drops downstream appear slightly smeared and distorted from perfect spheres because they were moving at rates faster than the capture rate of the camera ( 30 frames per second). We used oxazine 720 here for visual clarity of alternating droplet generation because its color was distinctly different from that of rhodamine 560 . The color of rhodamine 640 was very similar to (and indistinguishable from) that of rhodamine 560 in the movies recorded with our CCD camera. 
The width of the channels at the T-junctions was $20 \mu \mathrm{m}$. The main channel widened to $50 \mu \mathrm{m}$ downstream to ensure that the drops were close to spherical in shape, ${ }^{14}$ and that they did not contact the wall of the PDMS channel. The height of the channel was about $40 \mu \mathrm{m}$. We used drops with diameters between 20 and $40 \mu \mathrm{m}$. Bigger drops $(>80 \mu \mathrm{m})$ tend to deform and split into smaller drops at higher shear rates. We used frequencies of generation between $25 \mathrm{~Hz}$ and $3600 \mathrm{~Hz}$. The carrier fluid was driven by a syringe pump at fixed volumetric rates of flow. The disperse phase was driven by compressed nitrogen at fixed pressures. We found that pressure-driven flow for the disperse phase gave greater stability in the frequency of droplet formation than did active pumping using a syringe pump.

The drops contained solutions of dyes possessing absorption spectra that overlap at $532 \mathrm{~nm}$, and fluorescence spectra that differ significantly from each other. A single excitation source at $532 \mathrm{~nm}$ can therefore be used to pump multiple dyes to generate different emission wavelengths. We focus on rhodamine 560 and rhodamine 640 in this paper for the demonstration of principle because they are among the most efficient dyes commonly used for dye lasers. ${ }^{15,16}$ We used benzyl alcohol (refractive index $n_{\mathrm{D}}=1.54$, viscosity $\mu=8 \mathrm{mPa}$-s) as solvent because it has a higher index of refraction than many other liquids, and is a good solvent for the dyes: ${ }^{17}$ a higher contrast in index between the drop and its surrounding medium provides a better confinement of light inside the drop, and results in a higher optical quality factor and a lower threshold power needed to achieve lasing due to reduced radiative loss than drops possessing lower refractive indices. ${ }^{\mathbf{1 8}}$

We used HFE-7500 $\left(\mathrm{C}_{7} \mathrm{~F}_{15} \mathrm{OC}_{2} \mathrm{H}_{5}\right.$, refractive index $n_{\mathrm{D}}=1.29$, viscosity $\mu=1.24 \mathrm{mPa}-\mathrm{s}$ ) purchased from $3 \mathrm{M}$ as the continuous phase. We included $0.18 \%$ (by weight) of a proprietary fluorosurfactant supplied by Raindance Technologies to prevent coalescence of the drops. ${ }^{19}$ Other liquids, and other methods of generating (and sorting) drops can also be used, so long as the generation of drops is stable, and so long as the contrast in refractive index is high enough for lasing action in whispering gallery mode.

For optical excitation of the dyes, we used a pulsed, frequencydoubled Nd:YAG laser at $532 \mathrm{~nm}$ as the pump. Each droplet formed a micro-cavity for confinement of light emitted from the dyes $\left(n_{\mathrm{D} \text {, droplet }}=1.54 ; n_{\mathrm{D} \text {, carrier }}=1.29\right)$. In micro-spherical cavities, light is confined by total internal reflections at the interface between the microsphere and its surrounding medium, and is concentrated in the whispering gallery modes (WGMs). The positions (or wavelengths) of these modes are dependent on the geometry of the micro-cavity (or, the diameter of a spherical micro-cavity), and the contrast in the refractive index between the cavity and the surrounding medium only. ${ }^{18}$ Microdroplets, with their molecularly smooth surfaces and small sizes, are ideal optical microcavities, and have been used to make low-threshold lasers and ultrasensitive optical sensors. ${ }^{20}$ To characterize lasing from drops containing solutions of dyes, we varied the pump pulse energy from 0.01 to $100 \mathrm{~J}-\mathrm{cm}^{-2}$. The pulse width was about $20 \mathrm{~ns}$. The pump beam was focused at the microchannel with a beam diameter around $30 \mu \mathrm{m}$. In our setup, lasing light from the drops emitted in all directions. We collected the optical output from the drops using a $10 \times$ objective normal to the plane of the microchannel (in the z-direction). ${ }^{21}$ The light was coupled to a spectrometer or photodiodes for characterization.

We actively synchronized the drops with the pump pulses to avoid the problem that drops would be unevenly excited if we used pump pulses at a fixed repetition rate. See Supplementary Fig. S1 for details. $\dagger$
Fig. 2a shows the output intensities from $36 \mu \mathrm{m}$-droplets containing 5-mM solutions of rhodamine 560 ("R560") and rhodamine 640 ("R640") in benzyl alcohol as a function of input pulse energy. The threshold for lasing was about $1 \mu \mathrm{J}$ per pulse. Fig. $2 \mathrm{~b}, \mathrm{c}$ show the lasing spectra from these drops at an excitation pulse energy of $0.25 \mathrm{~mJ}$, superimposed on their broad fluorescence spectra at input energy below the lasing threshold. Lasing occurred in the longer wavelength regions of the emission spectra of the dyes, where absorption of light from the dyes was smaller. The drops supported multi-mode lasing at the excitation power we used. ${ }^{22}$ The emission from droplets of R560 ranged from $582 \mathrm{~nm}$ to $594 \mathrm{~nm}$; while that from droplets of R640 ranged from $670 \mathrm{~nm}$ to $690 \mathrm{~nm}$. Each mode had a full width at half maximum around $0.3 \mathrm{~nm}$. The resolution of the spectrometer was $0.1 \mathrm{~nm}$.

Fig. 3a shows the switching of lasing wavelengths as a function of time. We highlighted parts of the spectra corresponding to lasing from R560 in blue $(\sim 570-595 \mathrm{~nm})$, and that from R640 in red ( $\sim 665-695 \mathrm{~nm})$. The switching time between the two colors was about $30 \mathrm{~ms}$. The cluster of lasing peaks shifted in wavelength $\pm 2 \mathrm{~nm}$ from drop to drop (Fig. 3b). Detailed analysis of the mode structure is beyond the scope of this paper (also see p.5 of supplementary information for more discussion $\dagger$ ); but we believe this shift was due to slight changes in the radius of the drops. If we assume the polydispersity of droplets is $1 \%$ - a number typical of drops generated using $\mathrm{T}$-junctions or flow-focusing devices ${ }^{23}$ - the shift in emission wavelength for a given mode, $\frac{\Delta \lambda}{\lambda}=\frac{\Delta r}{r}$, where $\Delta \lambda$ and $\lambda$ are the shift in wavelength of emission, and the average wavelength of emission, and $\Delta r$ and $r$ are the shift in radius and the average radius of the drop, ${ }^{18}$ would be approximately $6 \mathrm{~nm}$ if we assume an average wavelength of $600 \mathrm{~nm}$. For applications where the laser is used as a source of illumination, this shift in wavelength is not important. The effects of thermal oscillation (causing surface ripples on the surface of
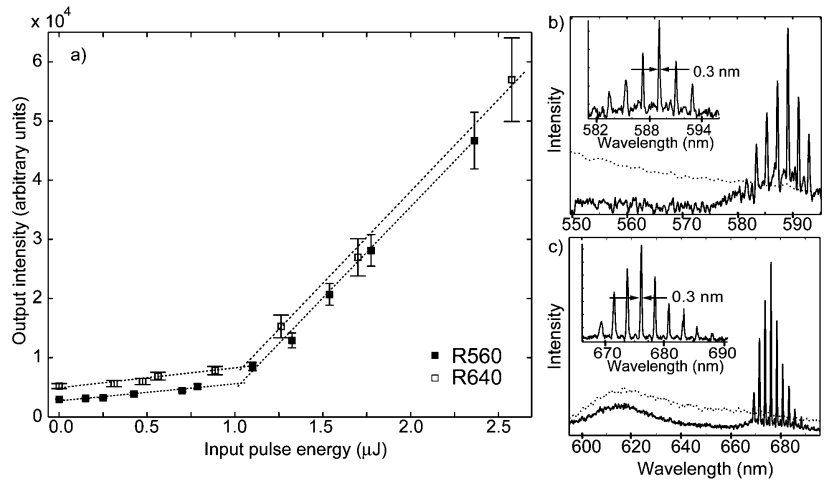

Fig. 2 a) Output intensity from $36 \mu \mathrm{m}$-droplets containing 5-mM solutions of rhodamine 560 and rhodamine 640 in benzyl alcohol as a function of input pulse energy. The points were averages of 7 sets of data. The bars represented standard deviations from the average. The dotted lines are guides to the eye only. b), c) show the lasing spectra of $36 \mu \mathrm{m}$-droplets containing $5-\mathrm{mM}$ solutions of rhodamine 560 and rhodamine 640 in benzyl alcohol respectively at input pulse energy of $0.25 \mathrm{~mJ} / \mathrm{pulse}$. The insets show magnified portions of these lasing emission spectra. The dotted lines show the fluorescence spectra (intensity scaled up about 10 times) from these drops at input energy below the lasing threshold. The diameter of the pump beam was about $30 \mu \mathrm{m}$. The resolution of the spectrometer was $0.1 \mathrm{~nm}$. 
a)

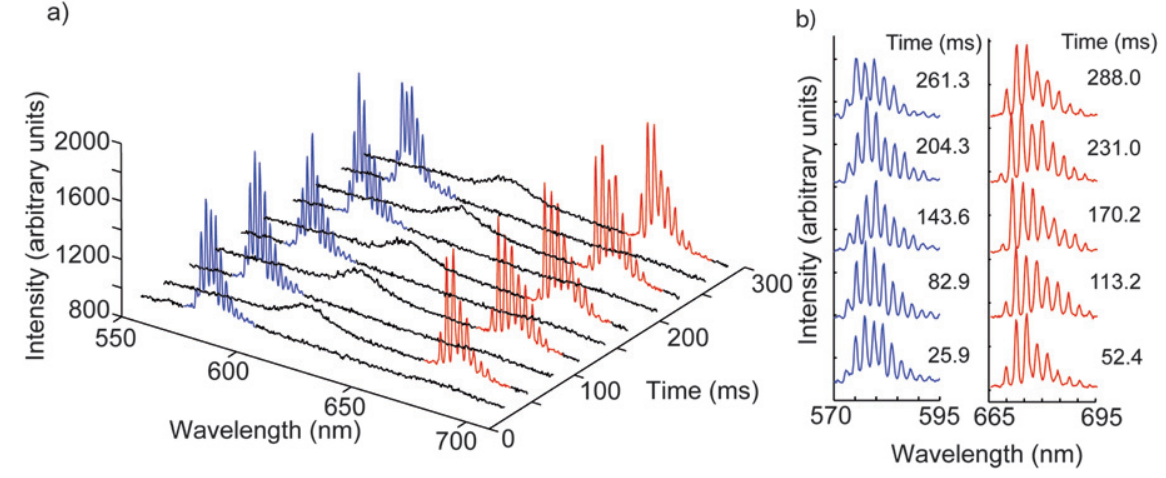

Fig. 3 a) shows the switching in lasing wavelengths from alternate drops ( $25 \mu \mathrm{m}$ in diameter) containing $5 \mathrm{mM}$ solutions of rhodamine 560 (highlighted in blue) and rhodamine 640 (highlighted in red) in benzyl alcohol as a function of time. b) shows the same spectra in a) overlaid on top of each other; this presentation shows the spectral shifts from droplet to droplet better than a) does. The rate of flow of the carrier fluid was $60 \mu \mathrm{L} \mathrm{h}{ }^{-1}$. The pressure applied to the disperse phase was 0.7 psi. The excitation pulse energy was $2.8 \mathrm{~mJ}$. The resolution of the spectrometer was $0.3 \mathrm{~nm}$.

the drops), and oscillations in the shape of the drop, were likely to be negligible at the rates of flow that we used..$^{18,24,25}$

Since the maximum acquisition rate of our spectrometer was limited to about $130 \mathrm{~Hz}$, we used two photodiodes (bandwidth up to $\mathrm{GHz}$ ) mounted with different filters ( $<600 \mathrm{~nm}$ for R560, and $>650 \mathrm{~nm}$ for R640) for characterization of the switching between the two lasing wavelengths at high speeds $(>130 \mathrm{~Hz})$. Fig. 4a shows the output from the two photodiodes normalized to the excitation intensity as a function of time. The switching time between the colors was about $0.85 \mathrm{~ms}$, corresponding to a switching speed of $1.18 \mathrm{kHz}^{26}$ We also acquired the lasing spectra separately (Fig. S2). $\dagger$ The excitation pulse energy was $1.1 \mathrm{~mJ}$.

We observed that $t_{1}$, the time to switch from R560 to R640 ( $t_{1} \sim$ $0.83 \mathrm{~ms}$ ), was always shorter than $\mathrm{t}_{2}$, the time to switch from R640 to $\mathrm{R} 560$ ( $\mathrm{t}_{2} \sim 0.86 \mathrm{~ms}$ ). Fig. $4 \mathrm{~b}$ shows a histogram for the distribution of different switching times for data recorded over 1.3 seconds (1558 drops). In movies taken with a fast camera (10 000 frames per second) (Fig. 4b insets), we observed that the drops moved in groups of two: $\mathrm{x}_{1}$, distance from a R560 drop to the following R640 drop, was slightly shorter than $\mathrm{x}_{2}$, distance from a R640 drop to the following R560 drop. This grouping originated from the generation of the drops at the T-junctions. The variation in the intensity of lasing (normalized to the pump intensity) was about 3.9\% for R560, and 9\% for R640, for data recorded for 1558 drops. While we are still investigating the origin of this variation in lasing intensity, we suspect it is due to small changes in the radius or speed of the drops.

The switching time between the lasing wavelengths is determined solely by the speed at which the drops pass through the optical excitation region; this speed depends on the rate of generation of the drops in the simple microfluidic design here. The alternation in the generation of the two types of drops from two opposing T-junctions has been characterized using the capillary number, $C a\left(C a=\frac{u_{c} \eta_{c}}{\gamma}\right.$, where $u_{c}$ and $\eta_{c}$ are the speed of flow and viscosity of the continuous phase, $\gamma$ is the interfacial tension between the drop and the continuous phase). ${ }^{12}$ Briefly, for $C a<0.001$, coalescence between the two disperse phases occurs. For $0.001<C a<0.05$, the drops break off in alternation in the squeeze regime. For $0.05<C a<0.13$, the drops still alternate in break-off. The break-off is in the dripping regime, however, and the size of the drops is smaller than the width of the main channel at the junction. For $\mathrm{Ca}>0.13$, shear dominates over
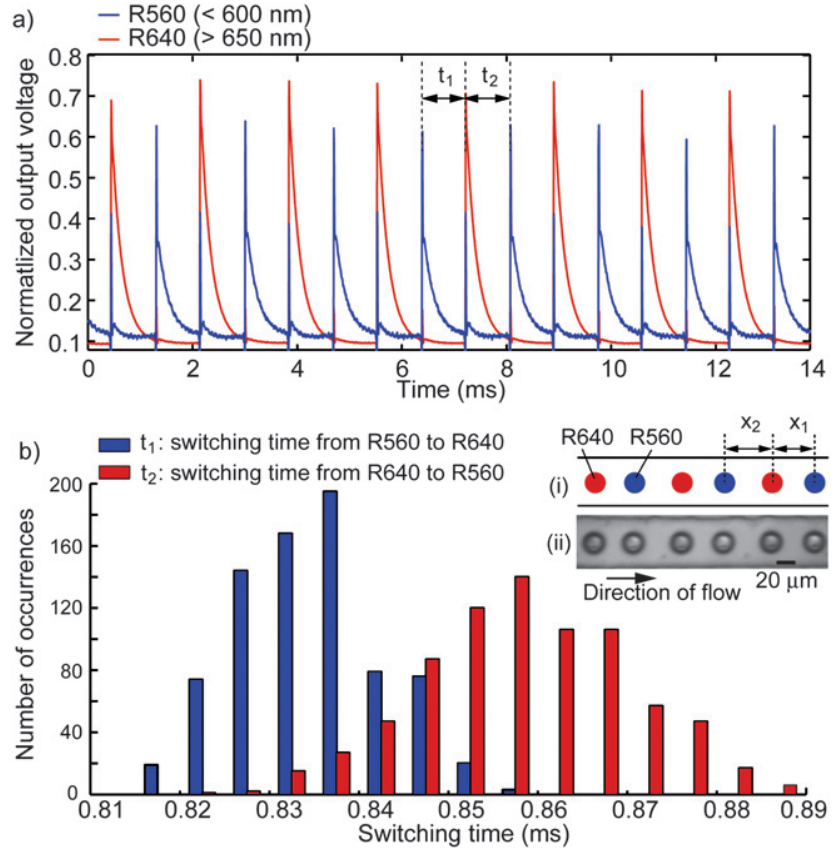

Fig. 4 a) Output voltage from photodiodes normalized to the pump intensity for wavelengths $<600 \mathrm{~nm}$ (corresponding to lasing of drops of rhodamine 560 "R560"), and $>650 \mathrm{~nm}$ (corresponding to lasing of drops of rhodamine 640 "R640") respectively as a function of time. The rate of flow of the carrier fluid was $200 \mu \mathrm{L} \mathrm{h}^{-1}$. The pressure applied to the disperse phase was 1 psi. b) shows a histogram showing the distribution of switching times between consecutive drops of R560 and R640. $t_{1}$ and $t_{2}$ are the times to switch from a R560 drop to a R640 drop, and vice versa. The bin size for the histogram is 20 . Inset (ii) shows a snapshot of a movie of the droplets flowing in the microchannel. Inset (i) shows the scheme for this snapshot. $\mathrm{x}_{1}$ and $\mathrm{x}_{2}$ are the distances from a R560 drop to the next R640 drop, and from a R640 drop to the next R560 drop respectively.

interfacial tension; instead of forming drops at the T-junctions, the fluids flow in parallel or laminar streams. We estimate a maximum switching rate of $2.2 \mathrm{kHz}$ (at $\mathrm{Ca}=0.05$ ) or $5.7 \mathrm{kHz}$ (at $\mathrm{Ca}=0.13$ ) for $20-\mu \mathrm{m}$ drops with the liquids and geometry of channel we used. ${ }^{27}$ Our system achieved a maximum rate of switching between the two types of drops at $3.63 \mathrm{kHz}$ (where the carrier fluid flowed at $400 \mu \mathrm{L} \mathrm{h}^{-1}$, and we applied 1 psi to the disperse phase) before the fluids started 
flowing in laminar streams. The generation of the drops was already in the dripping regime, however. The variation in the lasing intensity from the drops was larger ( $12 \%$ for R560, and $16 \%$ for R640); this larger variation was probably caused by a wider distribution in the size of the drops generated compared with the case when the generation was in the squeeze regime $(<2 \mathrm{kHz})$. It is possible to achieve higher speed of switching, by using liquids with a lower ratio of viscosity to interfacial tension $\left(\frac{\eta_{c}}{\gamma}\right)$, or by using smaller droplets or channels with smaller dimensions. The drawback of using even smaller drops is the increased radiative loss of light from the drops; ${ }^{18}$ a higher excitation power will be necessary to achieve lasing.

We have demonstrated a small, on-chip multi-color laser based on whispering-gallery-mode lasing from dye-containing droplets generated in a microfluidic channel. We have only shown the switching of two colors; switching among more colors should be possible using other geometries of droplet generators. ${ }^{28}$ Further broadening of the spectral range of our laser should also be possible by using UV excitation, and energy transfer between two or more dyes per drop, for example. Lasing from the drops is multi-modal; it has been shown, however, that single-mode lasing is possible using smaller drops, if high spectral purity is desired. ${ }^{2}$ Our current way of coupling light produced by lasing from the drops into the detector is not ideal, and although we have not measured the output power and efficiency of our system, we suspect it is very low. Better coupling techniques (such as incorporation of a tapered waveguide in the channel) ${ }^{29}$ should improve the optical output coupling efficiency.

The advantages of our system include its high rate of switching between different wavelengths - this high speed of switching (up to 3.6 $\mathrm{kHz}$ ) is made possible only in microfluidic droplet-based systems; and small volume of drops which allows low consumption rate of fluids $\left(<1 \mathrm{~mL} \mathrm{~h}^{-1}\right)$, and extended period of operation. Since each drop is excited once only, undesirable phenomena such as photobleaching can be avoided. The dyes in the drops are always isolated from the PDMS wall by the carrier fluid; this isolation prevents dye molecules from partitioning into PDMS and increasing the background fluorescence over time. Furthermore, since this droplet-based laser is formed inside microfluidic channels, cofabrication ${ }^{30}$ and pre-alignment of this light source to other functional fluidic elements is simple.

The primary limitation of this system is the need for a bulky external laser for the excitation of dyes. With higher contrast in the index of refraction between the droplets and the carrier fluid, and better coupling of light into and out of the droplets, the threshold of lasing should decrease further. It should be possible, then, to use a lower power on-chip semiconductor laser as a source of excitation.

Although the speed of switching between different colors of emission in our system is slow compared to semiconductor lasers ( $>\mathrm{GHz}$ ), it is higher than any existing dye lasers, and should meet the demands of applications that do not require fast switching, such as in-channel illumination for optical characterization of analytes or cells, ${ }^{31}$ and on-chip flow cytometry or fluorescence activated cell sorting that require multiple excitation wavelengths. Further modification of the current system can also be used for label-free, intra-cavity sensing by quenching of lasing or shifting the lasing wavelengths. ${ }^{32,33}$

\section{Acknowledgements}

This work was funded in part by DARPA award number W911NF07-10647, and the Center for Optofluidic Integration at Caltech under award HR011-04-1-0032 from DARPA. A portion of this work was supported by the Micro/Nano Fluidics Fundamentals Focus (MF3) Center at the University of California, Irvine. We thank Dr. Brian Hutchison, and Dr. Darren Link from Raindance Technologies for the supply of the fluorosurfactant. We also thank Drs Claudiu Stan, Ratmir Derda, Michinao Hashimoto, and Coskun Kocabas for helpful discussions.

\section{References}

1 M. Tanyeri, R. Perron and I. M. Kennedy, Opt. Lett., 2007, 32, 2529 2531.

2 J. Schafer, J. P. Mondia, R. Sharma, Z. H. Lu, A. S. Susha, A. L. Rogach and L. J. Wang, Nano Lett., 2008, 8, 1709-1712.

3 S. X. Qian, J. B. Snow, H. M. Tzeng and R. K. Chang, Science, 1986, 231, 486-488.

4 T. Thorsen, R. W. Roberts, F. H. Arnold and S. R. Quake, Phys. Rev. Lett., 2001, 86, 4163-4166.

5 P. Garstecki, I. Gitlin, W. DiLuzio, G. M. Whitesides, E. Kumacheva and H. A. Stone, Appl. Phys. Lett., 2004, 85, 2649-2651.

6 D. V. Vezenov, B. T. Mayers, R. S. Conroy, G. M. Whitesides, P. T. Snee, Y. Chan, D. G. Nocera and M. G. Bawendi, J. Am. Chem. Soc., 2005, 127, 8952-8953.

7 B. Bilenberg, T. Rasmussen, S. Balslev and A. Kristensen, J. Appl. Phys., 2006, 99, 023102.

8 Z. Li, Z. Zhang, A. Scherer and D. Psaltis, Opt. Express, 2006, 14, $10494-10499$.

9 J. Godin, C.-H. Chen, S. H. Cho, W. Qiao, F. Tsai and Y.-H. Lo, J. Biophotonics, 2008, 1, 355-376.

10 J. C. McDonald, D. C. Duffy, J. R. Anderson, D. T. Chiu, H. Wu, O. J. Schueller and G. M. Whitesides, Electrophoresis, 2000, 21, 27-40.

11 K. Ahn, C. Kerbage, T. P. Hunt, R. M. Westervelt, D. R. Link and D. A. Weitz, Appl. Phys. Lett., 2006, 88, 024104.

12 B. Zheng, J. D. Tice and R. F. Ismagilov, Anal. Chem., 2004, 76, 4977-4982.

13 L.-H. Hung, K. M. Choi, W.-Y. Tseng, Y.-C. Tan, K. J. Shea and A. P. Lee, Lab Chip, 2006, 6, 174-178.

14 Fluidic disks (with flat top and bottom) can also lase; the optical output from these drops would be concentrated in the $x-y$ plane (Fig. 1), however. Since we used an objective placed behind our device to collect light from the drops in the z-direction, we could not collect any lasing output efficiently if the drops were diskshaped. The spherical shape was therefore critical here, and the optical pump region was always downstream in the channel where the drops were close to spherical in shape.

15 The dyes we have tried that lased (using an excitation wavelength at $532 \mathrm{~nm}$ ) included: solutions of rhodamine 560, rhodamine 590, Kiton red, rhodamine 640 , cresyl violet, and oxazine 720 in benzyl alcohol

16 Dye Lasers, ed. F. P. Schaefer, Springer, 2nd Rev. Ed edn., 1977.

17 We have also tried (1:1 mixture of dimethyl sulfoxide and ethylene glycol (effective index $n_{\mathrm{D}}=1.45$ ), and $70 \%$ glycerol in water (effective index $n_{\mathrm{D}}=1.43$ )).

18 R. K. Chang and A. J. Campillo, Optical Processes in Microcavities, World Scientific Pub. Co. Inc., 1996.

19 C. Holtze, A. C. Rowat, J. J. Agresti, J. B. Hutchison, F. E. Angile, C. H. J. Schmitz, S. Koster, H. Duan, K. J. Humphry, R. A. Scanga, J. S. Johnson, D. Pisignano and D. A. Weitz, Lab Chip, 2008, 8, 1632-1639.

20 A. M. Armani, R. P. Kulkarni, S. E. Fraser, R. C. Flagan and K. J. Vahala, Science, 2007, 317, 783-787.

21 Other methods of coupling light from optical microspheres include using tapered waveguide or prism, ${ }^{29}$ in these cases most of the light would exit the microsphere from the coupling region into the waveguide or prism.

22 H. B. Lin, J. D. Eversole and A. J. Campillo, J. Opt. Soc. Am. B, 1992, 9, 43-50.

23 P. Garstecki, H. A. Stone and G. M. Whitesides, Phys. Rev. Lett., 2005, 94, 164501 .

24 H. M. Tzeng, M. B. Long, R. K. Chang and P. W. Barber, Opt. Express, 1985, 10, 209-211.

25 In movies taken using a fast camera, the drops were spherical and symmetrical at all conditions of flow we applied. Although we could 
not measure the exact shape of our drops given the resolution of our imaging system was only about 4 microns, we do not believe there was any source that would introduce systematic oscillations to the shape of the drops as a function of time.

26 The lifetime of the dye molecule's excited state should be about $5 \mathrm{~ns},{ }^{16}$ and the lifetime in the droplet cavity should be $<0.6$ ps. ${ }^{18}$ The pulse width of each droplet lasing peak in Fig. 4a, should therefore be limited by that of the pump pulse width $(\sim 20 \mathrm{~ns})$. The acquisition rate of our computer (using Labview FPGA module) was only $200 \mathrm{kHz}$ (i.e. time between each sampling was $5 \mu \mathrm{s}$ ), which was too low to sample the lasing pulses ( $20 \mathrm{~ns}$ long), in the form of voltage output from the photodiodes, directly. The circuit we used to amplify the voltage from the photodiodes introduced a resistivecapacitive (RC) decay time of about $300 \mu$ s to each spike in voltage. This relatively long decay time was sufficient for the computer to sample and acquire the lasing pulses at $200 \mathrm{kHz}$. It was also possible to use an oscilloscope (acquisition rate up to $10 \mathrm{GHz}$ ) to sample the lasing pulses. The maximum length of data that could be recorded was limited to a few microseconds, however; this made analysis of switching behavior between the drops for extended period of time difficult.
27 The viscosity of the continuous phase (HFE 7500) $\eta_{\mathrm{c}}=1.24 \mathrm{mPa}-\mathrm{s}$, and the interfacial tension (HFE 7500 with $0.18 \%$ surfactant, and benzyl alcohol) $\gamma=5.44 \mathrm{mN} / \mathrm{m}$. The speed of flow $u_{\mathrm{c}}$ at $C a=0.05$ is $0.22 \mathrm{~m} / \mathrm{s}$. Since we expanded the width of the channel to $50 \mu \mathrm{m}$ downstream, the speed of flow reduced to $0.09 \mathrm{~m} / \mathrm{s}$. If we assume the spacing between consecutive drops is roughly the same as the diameter of the drop $(20 \mu \mathrm{m})$, the switching time between consecutive drops is about $0.45 \mathrm{~ms}$, corresponding to a switching frequency of $2.2 \mathrm{kHz}$. The calculation is the same for $\mathrm{Ca}=0.13$.

28 M. Hashimoto, P. Garstecki and G. M. Whitesides, Small, 2007, 3, 1792-1802.

29 M. Cai, O. Painter and K. J. Vahala, Phys. Rev. Lett., 2000, 85, $74-77$.

30 A. C. Siegel, S. K. Y. Tang, C. A. Nijhuis, M. Hashimoto, S. T. Phillips, M. D. Dickey and G. M. Whitesides, Submitted.

31 J. Clausell-Tormos, D. Lieber, J.-C. Baret, A. El-Harrak, O. J. Miller, L. Frenz, J. Blouwolff, K. J. Humphry, S. Koester, H. Duan, C. Holtze, D. A. Weitz, A. D. Griffiths and C. A. Merten, Chem. Biol. (Cambridge, MA, U.S. ), 2008, 15, 427-437.

32 F. Vollmer and S. Arnold, Nat. Methods, 2008, 5, 591-596.

33 T. Melikhan and I. M. Kennedy, Sens. Lett., 2008, 6, 326-329. 